\title{
The Trajectory Simulation of Performance Test Manipulator
}

\author{
Jie Liu ${ }^{1, a^{*}}$, Hao Gao ${ }^{1, b}$, Fangyi Zong ${ }^{1, c}$ \\ ${ }^{1}$ Technology Center of Oceanographic Instrumentation Shandong, No.28 Zhejiang \\ road,Qingdao,China \\ aliujie0232@163.com, bogahao_tony@163.com, czongfangyi@163.com
}

Key words: manipulator; D-H method; kinematics; simulation; ADAMS

Abstract. Using D-H method develop HY-3 manipulator's trajectory and establish the kinematic model. Using mechanical system simulation software-ADAMS to simulate manipulator's trajectory and verify the correctness of theoretical analysis.

\section{Introduction}

HY-3 performance test manipulator is new type equipment used to appliance door hinge durability test. It can simulate the real situation of manpower open and close cabinet door with the high degree of automation and reliability. The manipulator appearance is shown in Fig1.
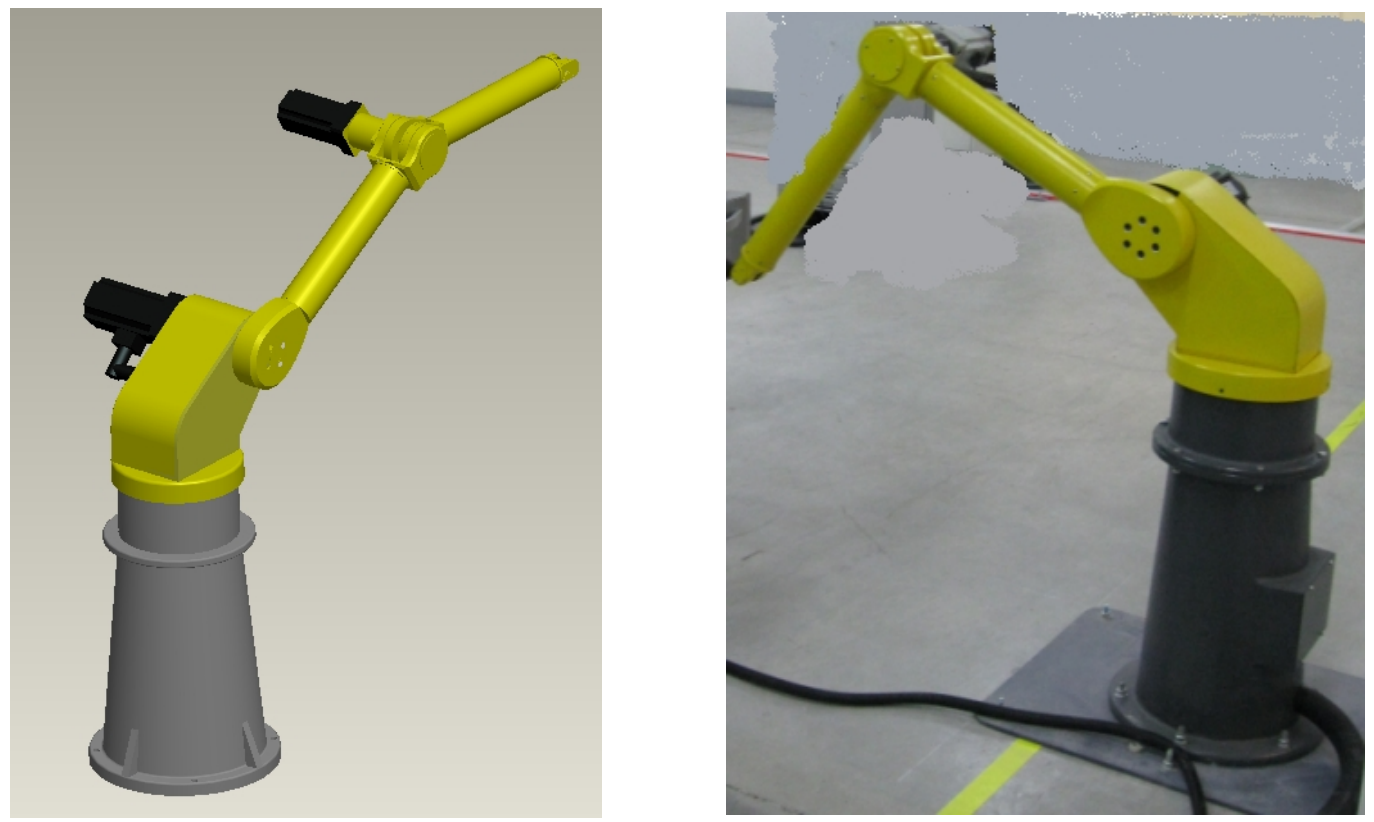

Fig.1 Outside drawing of HY-3 performance test manipulator

Appliance hinge life test has a high demand on manipulator's trajectory. But the manipulator is a strongly coupled and nonlinear system, difficult to establish a precise mathematical model. In this article, we use virtual technology to do the kinematics and dynamics simulation for HY-3 type manipulator, simulating the trajectory to improve performance of manipulator to meet the test requirements.

\section{Kinematics Model of Manipulator}

HY-3 type manipulator is composed by pedestal, arm and forearm, having 3 rotary joint. It is a 3-DOF manipulator. We often use a minimum degree of freedom which can meet the job 
requirements according to the requirements of practical tasks when we designing the manipulator. While the redundant freedom can make the manipulator has greater flexibility and obstacle avoidance capability, but also increases the difficulty of controlling.

Up to now, there are many domestic and foreign scholars have conducted research on manipulator motion control. Tie shi zhao and Jian S.Dai get the dynamics model and coupled constraint equation of manipulator by Jacobian Matrix and the Newton - Euler equation [1]. Tchon and Muszynski take into account the flexibility of a mobile manipulator in kinematics model [2]. He Yong derived joint reference velocity by direct differentiation inverse kinematics method for controlling the position of the manipulator [3]. In this paper, we use Denavit-Hartenberg method describe the relationship between the position and attitude of the manipulator.

The D-H method is commonly used in manipulator kinematics analysis.

We describe the spatial geometry of each link relative to reference coordinate system with homogeneous coordinates, and describe the spatial geometry of two adjacent links with $4 \times 4$ homogeneous transformation matrix [4]. Based on the above, we can derive the spatial position and orientation relationship between the ends of the manipulator coordinate system relative to the reference coordinate system.

Suppose each transformation matrix between the adjacent two joints is ${ }_{n}^{n-1} T$ [5], then the conversion between the end of the manipulator coordinate system and the reference coordinate system is ${ }_{n}^{0} T={ }_{1}^{0} T{ }_{2}^{1} T \mathrm{~L} \quad{ }_{n}^{n-1} T$ where the $\mathrm{n}$ is the number of joint.

According to the initial posture of manipulator and D-H coordinate distribution, we calculated structural parameters of each link and motion parameters of each joint in Table 1.

Table 1 Structural parameters and motion parameters

\begin{tabular}{|c|c|c|c|c|c|}
\hline Link $i$ & $a_{i-1}$ & $\alpha_{i-1}$ & $\theta_{i}$ & $d_{i}$ & $\begin{array}{c}\text { Variable range of } \\
\left.\text { joint angle }{ }^{\circ}\right]\end{array}$ \\
\hline 1 & $a_{1}$ & -90 & 0 & 0 & $-180 \sim 180$ \\
\hline 2 & $a_{2}$ & 0 & -90 & 0 & $-30 \sim 100$ \\
\hline 3 & 0 & -90 & 0 & 0 & $-110 \sim 110$ \\
\hline
\end{tabular}

According the link conversion formula:

$$
{ }_{i}^{i-1} T=\left[\begin{array}{cccc}
\cos \theta_{i} & -\sin \theta_{i} & 0 & a_{i-1} \\
\sin \theta_{i} \cos \alpha_{i-1} & \cos \theta_{i} \cos \alpha_{i-1} & -\sin \alpha_{i-1} & -d_{i} \sin \alpha_{i-1} \\
\sin \theta_{i} \sin \alpha_{i-1} & \cos \theta_{i} \sin \alpha_{i-1} & \cos \alpha_{i-1} & d_{i} \cos \alpha_{i-1} \\
0 & 0 & 0 & 1
\end{array}\right]
$$

We can get the transformation matrix of adjacent links: 


$$
\begin{aligned}
& { }_{1}^{0} T=\left[\begin{array}{cccc}
\cos \theta_{1} & -\sin \theta_{1} & 0 & 0 \\
\sin \theta_{1} & \cos \theta_{1} & 0 & 0 \\
0 & 0 & 1 & 0 \\
0 & 0 & 0 & 1
\end{array}\right] \\
& { }_{2}^{1} T=\left[\begin{array}{cccc}
\cos \theta_{2} & -\sin \theta_{2} & 0 & a_{1} \\
0 & 0 & 1 & 0 \\
-\sin \theta_{2} & -\cos \theta_{2} & 0 & 0 \\
0 & 0 & 0 & 1
\end{array}\right] \\
& { }_{3}^{2} T=\left[\begin{array}{cccc}
\cos \theta_{3} & -\sin \theta_{3} & 0 & a_{2} \\
\sin \theta_{3} & \cos \theta_{3} & 0 & 0 \\
0 & 0 & 1 & 0 \\
0 & 0 & 0 & 1
\end{array}\right]
\end{aligned}
$$

We can get the arm transformation matrix of manipulator by multiplying the above link transformation matrices:

$$
\begin{aligned}
{ }_{3}^{0} T={ }_{1}^{0} T_{2}^{1} T_{3}^{2} T & =\left[\begin{array}{cccc}
n_{x} & o_{x} & a_{x} & p_{x} \\
n_{y} & o_{y} & a_{y} & p_{y} \\
n_{z} & o_{z} & a_{z} & p_{z} \\
0 & 0 & 0 & 1
\end{array}\right] \\
n_{x} & =\cos \theta_{1} \cos \left(\theta_{2}+\theta_{3}\right) ; n_{y}=\sin \theta_{1} \cos \left(\theta_{2}+\theta_{3}\right) ; n_{z}=-\sin \left(\theta_{2}+\theta_{3}\right) ; \\
o_{x} & =-\cos \theta_{1} \sin \left(\theta_{2}+\theta_{3}\right) ; o_{y}=-\sin \theta_{1} \sin \left(\theta_{2}+\theta_{3}\right) ; o_{z}=-\cos \left(\theta_{2}+\theta_{3}\right) ; \\
a_{x} & =-\sin \theta_{1} ; a_{y}=\cos \theta_{1} ; a_{z}=0 ; \\
p_{x} & =\cos \theta_{1}\left(a_{1}+a_{2} \cos \theta_{2}\right) ; p_{y}=\sin \theta_{1}\left(a_{1}+a_{2} \cos \theta_{2}\right) ; p_{z}=-a_{2} \sin \theta_{2} .
\end{aligned}
$$

\section{Simulation Model and Analysis of Manipulator}

We use ADAMS software do the kinematics simulation of manipulator. ADAMS is a virtual prototype analysis software which developed by the Mechanical Dynamics Inc. ADAMS has powerful dynamics solver function, but it is relatively weak on solid modeling [6]. As the manipulator's complex structure, we build the solid model by Pro/E which is 3D modeling software, and then we import the model into ADAMS by interface file. So, the dimensional accuracy and positional accuracy of the model is ensured. We add the appropriate deputy campaign according to the movement form of the joint after the model is imported. Since all the joints of the manipulator are rotating joints, so we have to add rotation pair. 


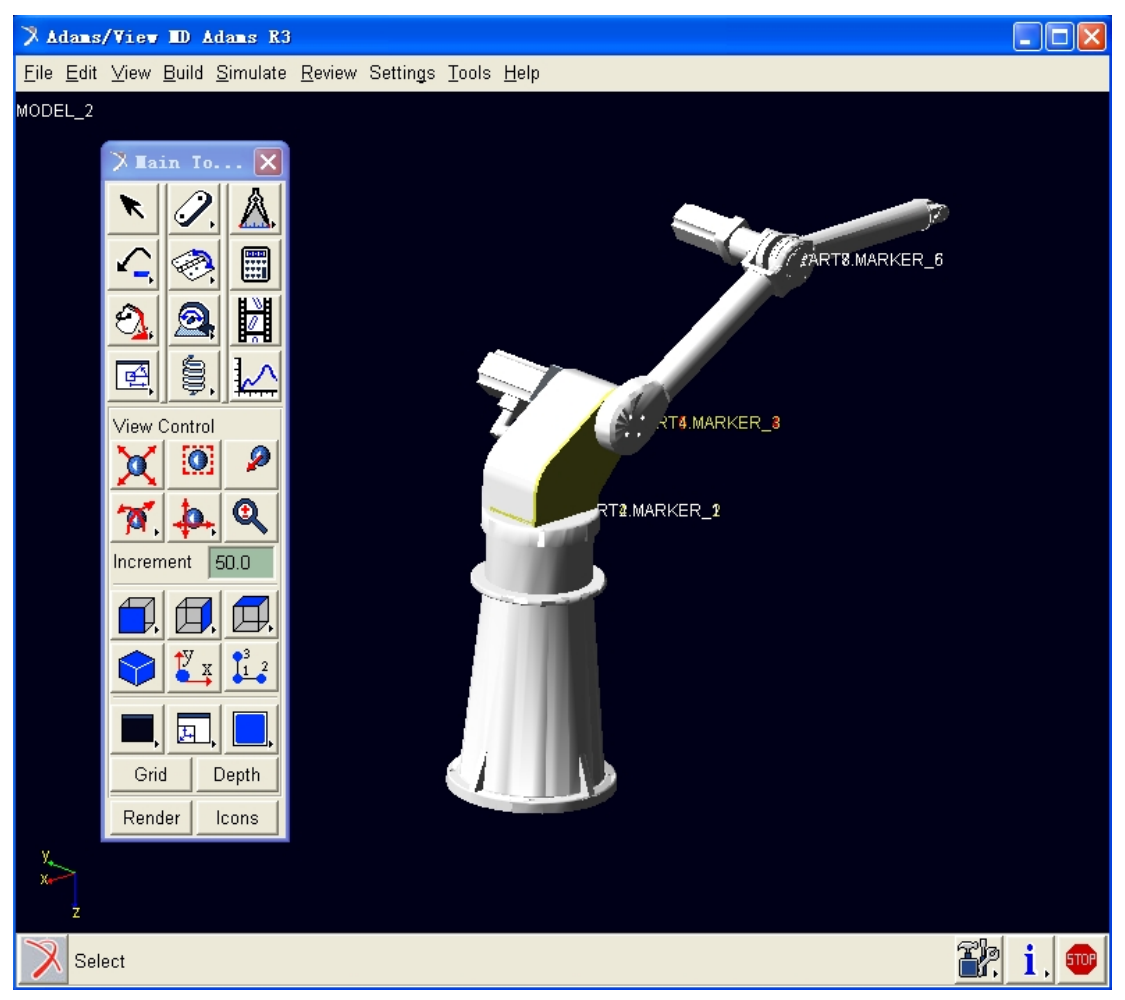

Fig.2 Model of manipulator in ADAMS

After the model is set up correctly, we can make a virtual sport analysis. The angular displacement of the joint as the independent variable, the simulation time is set to 5 seconds, the step size is set to 0.1 seconds.

Using the post-processing program of ADAMS, we can get the displacement, velocity and acceleration curve of the end of the manipulator's working point in the basis coordinate system.
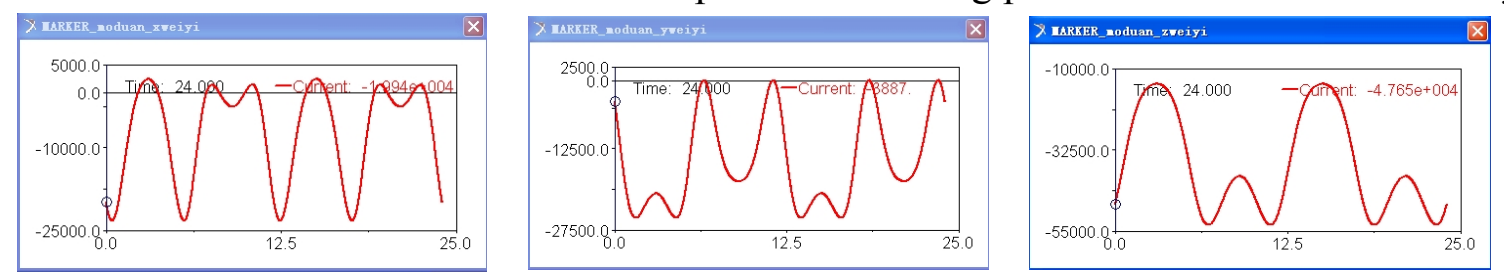

Fig.3 Chart of displacement of tail point
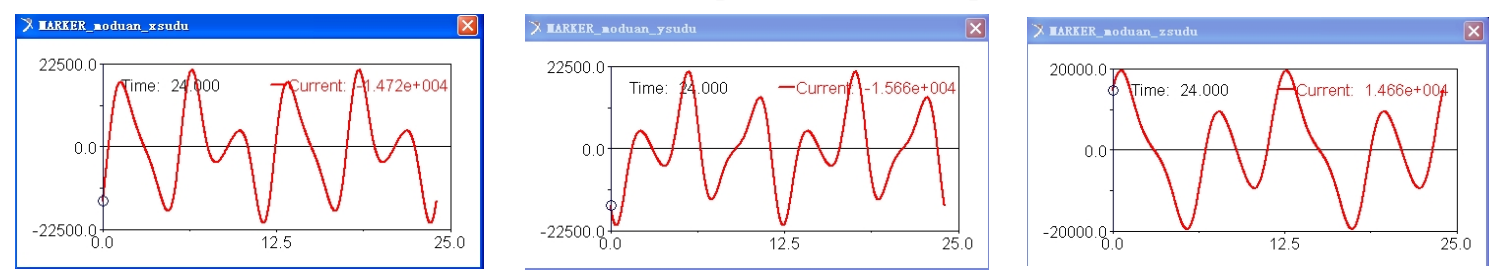

Fig.4 Chart of velocity of tail point
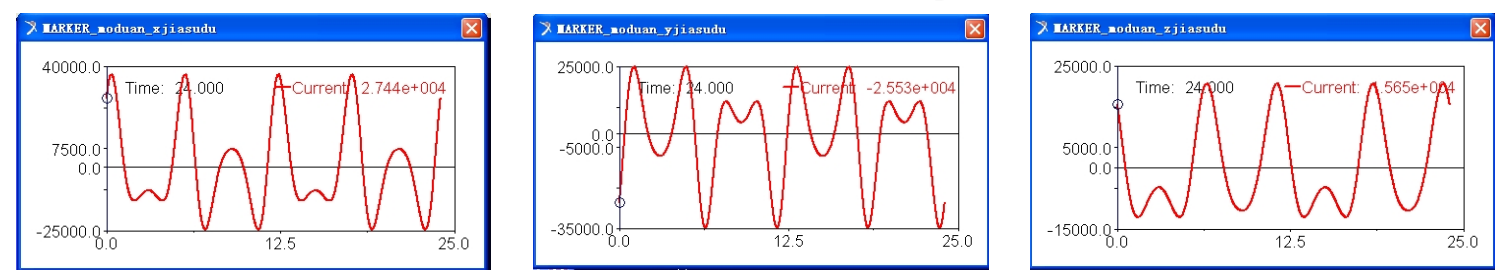

Fig.5 Chart of acceleration of tail point 


\section{Conclusion}

We can easily and quickly calculate the joint angle of a given end working point by DH method, and then planning a space trajectory. This method can simplify the calculation process of inverse kinematics with small amount of calculation. We can verified the theoretical calculations intuitive and easily by calculating and simulating the trajectory using ADAMS. The simulation results are similar with the theoretical calculation results and it shows the correctness of theoretical calculations. In addition, the use of virtual simulation technology can shorten the robot design cycles and can cost savings.

\section{References}

[1] T. S. Zhao, J. S. Dai. Dynamics and coupling actuation of elastic under actuated manipulators. Journal of Robotic Systems. 20(2003) 135-146.

[2] S. Lin. Robust and intellingent control of manipulators mobile. Toronto: Graduate Department of Mechanical and Industrial Engineering University of Toronto, 2001.

[3] Y. He, Q. Shen. The Position Control of Prosethema Manipulator with Five Degree of Freedom. Journal of Donghua University. 29(2003)48-51.

[4] Ani Luo, Jiatai Zhang, Heping Liu. Simulation of Five-Degree-of-Freedom Manipulator Using ADAMS. Computer Simulation. 22(2005)201-204.

[5] Min Zhang, Xiuhua Shi, Xiangdang Du, Rui Jia. Kinematic Analysis of a Three-DOF Manipulator. Machinery \& Electronics. 1(2005)65-67.

[6] Jun Li, Junwen Xing, Wenhao Qin. ADAMS Tutorial Examples. Beijing: Beijing Institute of Technology Press, 2002. 